Revista Iberoamericana de

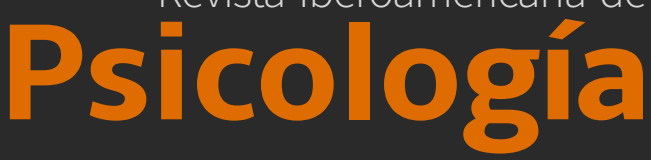

ISSN-I: 2027-1786 | e-ISSN: 2500-6517 Publicación Cuatrimestral

ID: 2027-1786.RIP.11307

Title:

Digital Inclusion and the Challenges To Educational Innovation in Brazil and Colombia

Título:Inclusión Digital y los Retos para la Innovación Educativa en Brasil y Colombia

Alt Title / Título alternativo:

[en]: Digital inclusion and the challenges to educational innovation in Brazil and Colombia

[es]: Inclusión digital y los retos para la innovación educativa en Brasil y Colombia

Author (s) / Autor (es):

Pereira Meireles da Silva, \& Díaz Gómez

Keywords / Palabras Clave:

[en]: $\quad$ Technological innovation; Digital divide; Learning activities; Education

[es]: Innovación tecnológica; Brecha digital; Actividades de aprendizaje; Educación

Proyecto / Project:

Experiencias subjetivas de aprendizaje mediadas por las TDIC de estudiantes universitarios en Brasil y Colombia

Financiación / Funding:

Experiencias subjetivas de aprendizaje mediadas por las TDIC de estudiantes universitarios en Brasil y Colombia

Submited:

2018-08-24

Acepted:

2018-10-31

\section{Resumen}

El trabajo tiene por finalidad discutir aspectos asociados a la inclusión digital en Brasil y Colombia, los retos educativos

que se plantean y, especialmente, los procesos de innovación educativa mediada por tecnologías digitales de información y comunicación (TDIC). Las reflexiones sobre

el tema se basan en el trabajo conducido en el marco de una investigación empírica sobre la influencia del nivel socioeconómico y las experiencias de aprendizaje mediadas por las tecnologías digitales en estudiantes universitarios brasileños y colombianos. Desde una perspectiva socioconstructivista

y situada del aprendizaje, el trabajo parte de tres convicciones. La primera considera

que las TDIC son importantes artefactos socioculturales mediadores de las prácticas sociales, configurando una nueva ecología del

aprendizaje. La segunda refiere al hecho de que, en la sociedad de la información (SI), las condiciones de acceso y uso de las TDIC no se dan igualmente para todos, sino que deben analizarse teniendo en cuenta la influencia del poder económico de los diferentes países y el nivel socioeconómico de las personas.

La tercera, es que las diversas experiencias de acceso y uso de las TDIC por las personas repercuten sobre las oportunidades educativas, restringiéndolas o ampliándolas

y llevando a la construcción de diferentes rutas de aprendizaje. En este sentido, desde los aspectos de la inclusión digital en Brasil

y Colombia, se discuten los retos que se plantean a la educación, especialmente a los procesos de innovación educativa.

\section{Abstract}

This paper aims to discuss the aspects of digital inclusion in Brazil and Colombia, and the challenges to education and, especially, the processes of educational innovation mediated

by digital information and communication

technologies (DICT). The reflections about of subject are based on the work conducted

in the mark of a larger empiric research on influence of socioeconomic level and learning

experiences of Brazilian and Colombian

undergraduates DICT-mediated. From a situated and sociocultural approach, this paper is based on three convictions. First considers

that the DICT are important sociocultural

artefacts that mediate the social practices,

configuring a new ecology of learning. Second refers to the fact that, in information society, the conditions of access and use of the DITC are not equally for all, taking into account the influence of the economic power of different

countries and socioeconomic levels of the people. Third conviction is that the different people experiences of access and use of DICT reverberate on the educational opportunities,

restricting or expanding them and leading

to the construction of different learning trajectories. In this sense, from the aspects of digital inclusion in Brazil and Colombia, the challenges that are posed to education are analyzed, especially the processes of educational innovation.

\section{Citar como:}

Pereira Meireles da Silva, A. M., \& Díaz Gómez, D. A. (2018). Inclusión Digital y los Retos para la Innovación Educativa en Brasil y Colombia. Revista Iberoamericana de Psicología issn-l:2027-1786, 11 (3), 81-88. Obtenido de: https://revistas.iberoamericana.edu.co/index.php/ripsicologia/article/view/1450-5072

Dra Algeless Milka Pereira Meireles da Silva, Msc Psi

Research ID: H-2508-2018

ORCID: https://orcid.org/0000-0002-1883-1916

Source | Filiacion:

Universidade Federal do Piauí

$\mathrm{BIO}$ :

Docente e investigadora de la carrera de Psicología de la Universidad Federal de Piauí, Brasil. Formadora del Programa Nacional de Formación de Profesores dela Educación Básica (PARFOR),Brasil. Coordinadora del Laboratorio de Psicología e Innovación Educativa (LAPSIN). Gestora de proyectos de investigación en colaboración internacional. Jurado de proyectos de investigación en Psicología a nivel de Maestría.

City | Ciudad:

Parnaíba [br]

e-mail:

milkameireles@ufpi.edu.br
Darwin Andrés Díaz Gómez, Msc Psi

Research ID: W-8990-2018

ORCID: https://orcid.org/0000-0002-6259-9122

Source | Filiacion:

Universidad de Cundinamrca

$\mathrm{BIO}$

Decano(EF) de la Facultad de Ciencias Sociales, Humanidades y Ciencias Políticas de la Universidad de Cundinamarca, Colombia.Docente, director y jurado de proyectos de investigación en Educación y Tecnologías de la Información y la Comunicación a nivel de Maestría. Miembro colaborador extranjero del Laboratorio de Psicología e Innovación Educativa (LAPSIN)de la Universidad Federal de Piauí ,Brasil.

City | Ciudad:

Fusagasugá [co]

e-mail:

darwinadiaz@ucundinamarca.edu.co 


\title{
Inclusión Digital y los Retos para la Innovación Educativa en Brasil y Colombia
}

\section{Digital Inclusion and the Challenges To Educational Innovation in Brazil and Colombia}

\author{
Algeless Milka Pereira Meireles da Silva \\ Darwin Andrés Díaz Gómez
}

\section{Introducción}

Este estudio hace parte una investigación más amplia realizada en el marco de un proyecto de cooperación internacional entre dos universidades públicas, una brasileña y la otra colombiana, sobre el uso de las tecnologías digitales de la información y comunicación (TDIC) por parte de estudiantes universitarios en actividades cotidianas, sus experiencias de aprendizaje y su relación con el nivel socioeconómico.

En este marco, el presente trabajo surge como fruto de los esfuerzos emprendidos a lo largo del desarrollo del proyecto en el sentido de comprender cómo la relación entre la educación y la inclusión digital ha sido abordada en la literatura y se ha constituido en objeto de estudio especialmente en Brasil y Colombia. Así, el trabajo tiene por objetivo discutir los aspectos de la inclusión digital en Brasil y Colombia y los retos que se plantean a la educación, especialmente, con relación a los procesos de innovación educativa en el contexto de ambos países. En efecto, el estudio pretende contribuir con el debate acerca de cómo las diferentes realidades de las personas respecto al acceso y uso de las TDIC afectan las oportunidades educativas de la población y demandan la planeación de acciones proficuas por parte del poder público en el sentido de garantizar la equidad.

Partiendo de una perspectiva socioconstructivista y situada del aprendizaje, consideramos las TDIC como importantes artefactos culturales mediadores de las prácticas sociales, que permiten generar y potencializar procesos de aprendizaje en los diferentes contextos de actividad transitados por las personas. En ese aspecto, el amplio uso de las TDIC en el actual contexto socio-histórico-cultural fomenta reflexiones sobre la función social de la educación y de las instituciones

educativas. Entre las reflexiones, se destaca el debate acerca de lo que significa aprender y desenvolverse como ciudadano capaz de seguir aprendiendo a lo largo de la vida en el contexto de la nueva ecología del aprendizaje (Coll, 2013).

Así las cosas, estudiar los procesos educativos en el ámbito de la nueva ecología del aprendizaje significa reconocer las diversas actividades cotidianas mediadas por las TDIC como potencialmente generadoras de oportunidades para aprender. En ese continuum, se resalta que desde la mirada teórica asumida en el trabajo, el aprendizaje ocurre en la medida que el sujeto transita por los diversos entornos, dentro y fuera de los contextos formales de educación, haciendo uso de los recursos disponibles e interactuando con los diferentes agentes educativos. Así, el protagonismo antes ocupado por las prácticas educativas llevadas a cabo en las instituciones responsables por el saber formal es asumido por la capacidad del sujeto en gestionar su propio proceso de aprendizaje. Esa capacidad se observa en la medida en que utiliza de manera eficiente los recursos ofrecidos por los diversos entornos, potencia su conocimiento y desarrolla habilidades y comportamientos más allá de los contenidos académicos.

No obstante, entre los beneficios impulsados por el desarrollo tecnológico y el amplio uso de las TDIC en las entrelíneas de la Sociedad de la Información (SI), se sitúa también la enorme brecha digital construida históricamente y observada entre los diferentes países o regiones dentro de un mismo país, poniendo el acento sobre la disparidad que se da especialmente en función de sus respectivos potenciales económicos (Greene, 2016; Selwyn, 2004). De ahí que, desde la perspectiva sociocultural (Anderson, Reder, \& Simon, 1996; Brown, Collins, \& Duguid, 1989; Lave \& Wenger, 1991; Leontiev, 1974; Roth \& Jornet, 2013), pensar el aprendizaje y los procesos educativos 


\section{Inclusión Digital y los Retos para la Innovación Educativa en Brasil y Colombia}

en la sociedad de la información requiere una mirada cuidadosa sobre el contexto histórico y cultural en que fue constituida.

A partir de lo anterior, a continuación presentamos algunas reflexiones sobre la constitución de la sociedad de la información con el objetivo de contextualizar la brecha digital que existe actualmente y que restringe las posibilidades de acceso a las TDIC, afectando así las oportunidades educativas de las personas y los procesos de innovación educativa.

\section{Los antecedentes de la brecha digital en la sociedad de la información}

La inclusión digital como vía para democratizar el acceso de las personas a las TDIC es un tema especialmente relevante en el marco de la sociedad de la información (SI). Así, si por un lado, la SI es comprendida como una sociedad centrada en la producción y en el consumo del conocimiento y de la innovación más que en los productos materiales (Robles, Marín, \& Zambrano, 2012), por otro, la inclusión digital se revela como un derecho humano clave para la equidad y el ejercicio de la ciudadanía (Bustamante, 2007).

De hecho, en el marco de la sociedad de la información, la amplia presencia de las tecnologías en los varios ámbitos de la vida social ayuda a reconfigurar las condiciones de existencia de las personas e instituciones. En ese aspecto, la discusión que proponemos parte del paradigma de la tecnología de la información que sitúa la revolución tecnológica como el punto de inflexión de los principales cambios en las prácticas sociales de los últimos tiempos, suplantando incluso, los generados por la revolución industrial (Castells, 2000).

La revolución tecnológica observada a partir de los años 70 fue liderada por los Estados Unidos y luego se expandió en los países con reconocido poder económico (Hasebrink, Jensen, Bulck, Hólig, \& Maeseele, 2015). En este aspecto, resaltamos que son múltiples los factores que se asocian al histórico de desarrollo tecnológico de los diversos países y entendemos que los impactos de la amplia presencia de las TDIC en la vida de las personas se dan de maneras diferentes en función de las diferencias sociales. De hecho, se observan discrepancias entre el potencial económico de los países y la repercusión sobre sus respectivas capacidades para producir y consumir la información y las tecnologías en general.

En efecto, es razonable pensar que dichas discrepancias no solo generan rutas diferentes de apropiación de las TDIC en las prácticas sociales que tienen lugar en los diferentes países, sino que potencializan situaciones de dependencia económica entre ellos. De ese modo, las relaciones de poder que se establecen tienden a perpetuar las condiciones de pobreza de las personas, especialmente en los países menos desarrollados (Castells \& Laserna, 1989; Hasebrink, Jensen, Bulck, Hólig, \& Maeseele, 2015).

Estudios diversos indican que el acceso y uso de las TDIC entre la población en general son influenciados por variables como el nivel socioeconómico, escolaridad, localidad dónde vive (zona rural o urbana), etnia, religión, entre otras (Comitê Gestor da Internet no Brasil., 2016; Comitê Gestor da Internet no Brasil., 2017; Greene, 2016; Instituto Nacional de Geografía Estadística., 2017; Observatorio Nacional de las Telecomunicaciones y de la Sociedad de la Información., 2016; Observatorio Nacional de las Telecomunicaciones y de la Sociedad de la Información., 2017). Así, los diferentes perfiles de acceso y uso de las TDIC en función de las más diversas variables repercuten sobre las experiencias subjetivas de aprendizaje que son generadas a través de la participación de las personas en las actividades cotidianas mediadas por las TDIC (Silva, 2017).

En el contexto de la SI, el poder está vinculado, entre otros aspectos, al acceso, manejo y producción de información, lo que ha llevado a la clasificación de los países como inforricos e infopobres, teniendo en cuenta el proceso y el ritmo de incorporación de las tecnologías (Cebrián, 1998). De hecho, pensar la ciudadanía y los derechos humanos en su sentido pleno y global requiere repensar los aspectos de la inclusión digital de las personas que viven en los diferentes países (Bustamante, 2007; López \& Samek, 2009). Estos aspectos fomentan el debate sobre la integración de la inclusión digital como tema a ser considerado en el ámbito de los derechos humanos, que, entre otros aspectos, parte del reconocimiento de la función de las TDIC como herramientas de apoyo al desarrollo educativo y comunitario.

\section{Inclusión digital en Brasil y Colombia}

Uno de los aspectos que consideramos básicos para discutir la inclusión digital corresponde al acceso que tienen los ciudadanos a las TDIC y, en función de eso, las políticas desarrolladas por las varias esferas del poder público como estrategia para disminuir la brecha digital. En niveles más profundos de la discusión, es fundamental observar, por ejemplo, en qué medida el acceso y uso de las TDIC, de hecho, acarrean beneficios efectivos a la población en términos de desarrollo comunitario y disminución de las desigualdades. En el presente trabajo, nos interesa particularmente abordar la inclusión digital teniendo en cuenta sus posibles efectos y los retos que se plantean a la educación y a la innovación educativa en el contexto brasileño y colombiano.

En lo que concierne al acceso de las personas a las TDIC, cerca del $67 \%$ de la población de América Latina y el Caribe están conectados a la red Internet, lo que significa decir que los demás 300 millones de personas no tienen acceso a red Miniwatts Marketing Group (2018). Se estima que el índice de personas conectadas a Internet en Brasil está por encima de la media de América Latina y el Caribe, alcanzando actualmente el $\mathbf{7 0 , 7 \%}$, mientras que en Colombia se observa el $63,2 \%$ de la población. De todas maneras, los índices de usuarios de Internet en ambos países se encuentran muy por debajo de la media de los países desarrollados, que llega al $\mathbf{9 5 \%}$ de la población en Norte América y al 85,2\% en Europa (Miniwatts Marketing Group., 2018).

Del total de la población que está conectada en los países de América Latina y el Caribe, sólo el $\mathbf{2 0 \%}$ de ella tiene acceso a banda ancha fija y cerca del $\mathbf{5 0 \%}$ a banda ancha móvil. Comparados los demás países, Brasil ocupa la tercera posición respecto al acceso de la población a la banda ancha móvil y la sexta en relación a la banda ancha fija, mientras Colombia, mantiene la octava posición en ambas situaciones (Comitê Gestor da Internet no Brasil., 2016).

A pesar de que Brasil y Colombia se posicionen entre los ocho primeros lugares en el ranking de los 27 países que componen el área de la América Latina y el Caribe, los índices de conectividad por banda ancha son muy inferiores a los observados en países como los de la Unión Europea. De hecho, los datos del último informe publicado por la ONTSI (2017) muestran que, en ellos, cerca del $\mathbf{8 3} \%$ de los hogares están conectados a Internet por medio de la conexión de banda ancha fija, mientras la conexión por banda ancha móvil alcanza hasta el $83,9 \%$. 
Respecto a la penetración de las TDIC entre la población, especialmente el móvil y los ordenadores, se constata que Brasil y Colombia presentan números similares y comparten el histórico de crecimiento en la última década. Considerándose, por ejemplo, la realidad brasileña, los números indican que alrededor del $\mathbf{8 6 \%}$ de la población brasileña utiliza móviles y cerca del $\mathbf{8 7 \%}$ de usuarios de internet en Brasil, accede a la red exclusivamente por medio de ellos. Asimismo, los ordenadores están presentes en el $\mathbf{5 0 \%}$ de los hogares brasileños, lo que significa el doble en comparación a la década anterior (Comitê Gestor da Internet no Brasil., 2016).

A pesar del panorama de crecimiento del número de usuarios de Internet y de acceso de la población a los diversos dispositivos TDIC en ambos países, aun se observa la brecha digital que hay entre Brasil, Colombia y los países desarrollados. Asimismo, los números, más allá de situarse por debajo de los índices de los países desarrollados, también no son representativos de la realidad de todas las personas que viven en las diversas regiones y pertenecen a los varios segmentos sociales.

Por lo anterior, se resalta que la brecha digital que se observa es influenciada por varios factores, entre ellos: el nivel socioeconómico de las personas, la región en dónde viven, el nivel de escolaridad, la edad y el género, para citar solo algunos (Comitê Gestor da Internet no Brasil., 2016). Considerando la realidad especialmente de Brasil y Colombia, observamos que los menores índices de acceso y uso de Internet y las TDIC en general, son encontrados entre las personas de los niveles socioeconómicos más bajos y que viven en regiones con dificultades históricas en cuanto al desarrollo económico y humano. Asimismo, los índices tienden a caer todavía más si observamos los datos que refieren a las personas que poseen los menores niveles de escolaridad y que viven en las zonas rurales.

\section{Inclusión digital y educación en Brasil y Colombia}

En general, los estudios conducidos en el contexto brasileño y colombiano sobre inclusión digital y educación abordan el desarrollo de herramientas, la proposición de métodos o estrategias para promocionar el uso de los diversos dispositivos TDIC por grupos específicos, como los estudiantes, las personas mayores, los jóvenes en situación de vulnerabilidad social, entre otros. En este aspecto, se destaca el interés de los investigadores por el análisis de programas de inclusión digital gubernamentales o llevados a cabo por entidades del tercer sector (Gonçalves, y otros, 2017; González \& Sánchez, 2013; Silva., 2011).

En los trabajos publicados, la relación entre inclusión digital y educación no es tratada de manera directa siendo, en general, observada en paralelo a la discusión de otras temáticas como ciudadanía, equidad, ética, salud, bien-estar, ocio, etc. (Moraes, Veiga, Vasconcellos, \& Santos, 2009; Pinho-Neto, 2014; Rashid, 2016). Es decir, son pocos los estudios que han enfocado el rol de la escuela o la universidad respecto a la inclusión digital de las personas en la sociedad (Siqueira \& Rothberg, 2014; Guimarães, Ribeiro, Echeveste, \& Jacques, 2013; Said-Hung, y otros, 2015; Silva., 2011). Entre las investigaciones que abordan explícitamente esta relación, desde el referencial teórico que orienta el presente trabajo, identificamos tres maneras distintas de comprenderla que, a nuestro juicio, posee diferentes implicaciones sobre las prácticas educativas y las políticas de apoyo a la innovación educativa.
La primera de ellas corresponde a la idea de la educación como requisito para la inclusión digital. Eso significa decir, que el bajo nivel de escolaridad es mencionado como un elemento relevante que tiende a dificultar la inclusión digital (Fernandes, Spagnuolo, \& Nascimento, 2017; Gonçalves, y otros, 2017; Santana \& Raymundo, 2014). En general, son estudios que no tienen como finalidad directa utilizar las TDIC en procesos educativos formales, sino que desarrollan proyectos para promocionar la inclusión digital a través del manejo de dispositivos o softwares para impulsar la autonomía, el empoderamiento y la ciudadanía en un público determinado, como los grupos de personas mayores, jóvenes en situación de vulnerabilidad social, profesionales de la salud, etc. Así, los niveles diferentes de escolaridad de las personas son comprendidos como una dificultad para el éxito pleno de la inclusión digital de los grupos y no es enfatizada directamente la potencialidad de las mismas TDIC como herramientas para seguir aprendiendo a lo largo y a lo ancho de la vida.

La segunda concepción que identificamos es la que comprende la educación como promotora de la inclusión digital (Paixão, y otros, 2011; Silva, Jambeiro, Lima, \& Brandão, 2005). En general, los estudios abordan la inclusión digital desde una perspectiva interdisciplinar, entrelazando conceptos como tecnología, educación, ética, ciudadanía, desarrollo social, entre otros. Asimismo, se tratan de investigaciones conducidas con públicos variados como comunidades rurales, profesionales de la salud que actúan en la atención primaria y estudiantes, en algunos casos habiendo comparación entre la realidad de Brasil y Colombia en lo que toca a las políticas públicas de apoyo. De hecho, se considera que hay una fuerte relación entre la inclusión digital y la educación, especialmente la que se da en los contextos formales, destacando sus efectos sobre la formación ciudadana. Así, se destaca el papel de las escuelas, universidades y organizaciones no gubernamentales como instituciones responsables por la enseñanza de contenidos y desarrollo de competencias digitales, sea como parte del currículo escolar, sea por medio de la acción de proyectos educativos de formación complementar (Arevalo \& Gamboa, 2015; Beltran, 2016; Cuevas-Cerveró \& García-Moreno, 2010; Martelo, Jiménez, \& Jaimes, 2017).

La tercera concepción observada refiere a la educación como consecuencia de la inclusión digital; es decir, la experiencia de acceso y uso de las TDIC es comprendida como algo potencialmente capaz de generar aprendizaje, teniendo en cuenta que amplían las oportunidades educativas dentro y fuera de los contextos formales (Moraes, Veiga, Vasconcellos, \& Santos, 2009; Pinho-Neto, 2012). En este sentido, los estudios, en general, comparten los resultados de proyectos de inclusión digital que tienen como foco propiciar el acceso, uso y apropiación de las TDIC por grupos considerados en situación de vulnerabilidad social, como las personas que trabajan en los servicios de limpieza urbana y la población carcelaria, para ampliar las oportunidades educativas, especialmente, las de formación profesional. La idea central es que por medio de las herramientas tecnológicas desarrollen las competencias para acceder, manejar y actuar sobre la información, disfruten de procesos educativos que lleven a mejorar el ejercicio de la ciudadanía.

En el presente trabajo, se asume una mirada dialéctica acerca de la relación entre la inclusión digital y la educación. Por un lado, hay que reconocer la importancia de la escolaridad como variable que influye sobre el acceso y uso de las TDIC en los diferentes países. Sin embargo, ponemos el acento sobre la importancia de crear e implementar políticas de apoyo al uso de las tecnologías como manera de sacar provecho de su potencialidad para mediar procesos educativos, especialmente los acreditados por instituciones formales, teniendo en cuenta, mejorar los niveles de escolaridad de la población. 


\section{Inclusión Digital y los Retos para la Innovación Educativa en Brasil y Colombia}

Con base en todo lo discutido hasta el momento, conviene reflexionar sobre los retos que se plantean a la educación y a los procesos de innovación educativa en ambos países desde la realidad de la (no o baja) inclusión digital que se observa.

\section{Los retos para la innovación educativa en Brasil y Colombia}

En conformidad con lo anterior, reiteramos que es fundamental pensar la inclusión digital a partir de los aspectos que consideramos básicos como el acceso, el uso y la apropiación de las TDIC, que se observan diferencialmente en personas y comunidades (Vega, 2016). Así, la inclusión digital debe considerar no solo el acceso y manejo de los dispositivos, sino aspectos como la calidad de la conexión a Internet y otros factores que dificultan el uso de las tecnologías como motor de cambio social e igualdad de derechos.

De ese modo, resaltamos la necesidad de profundizar la cuestión con el propósito de superar la idea de que la inclusión digital se restringe a la simple presencia de las TDIC en la vida de las personas. Ponemos de manifiesto que el uso de las TDIC en los procesos de enseñanza y aprendizaje tampoco es suficiente para garantizar la innovación educativa. Asumimos la innovación educativa como una acción deliberada que, más allá de las tecnologías, incorpora la interacción entre personasy el desarrollo de procesos. Así, partiendo de una mirada socioconstructivista y situada, consideramos la innovación educativa como un motor propulsor de cambios significativos y ajustados en los procesos y en los resultados de aprendizaje, lo que pone en relieve no solo la idea de innovar, sino de transformar la manera de enseñar y aprender.

El primer reto que mencionamos trata del hecho de que pensar la educación en el actual contexto pasa por re-discutir los paradigmas en los que están pautados los sistemas educativos, teniendo en cuenta las necesidades sociales que emergen en el nuevo escenario que caracteriza el siglo XXI. Así, si pensamos a nivel de sistema educativo, podemos mencionar la necesidad de ajustar las competencias curriculares en respuesta a las necesidades sociales que emergen en el siglo XXI (Coll, 2013).

Como se puede suponer, el ajuste del currículo escolar debe involucrar la discusión sobre cuestiones estructurantes que orientan el sistema educativo, que son las finalidades, objetivos y metas que se pretenden alcanzar con la conclusión del proceso de escolarización formal. Además de eso, es fundamental debatir sobre los conceptos de educación, aprendizaje y formación ciudadana que parecen razonables para fundamentar las prácticas educativas innovadoras, que promocionen la inclusión digital.

Si pensamos en nivel de escuela, la discusión sobre los paradigmas educativos en frente al nuevo escenario igual se hace fundamental, especialmente, teniendo en cuenta los ajustes curriculares con base al contexto local y las necesidades de los varios actores que constituyen la comunidad escolar. En ese sentido, respecto a los impactos de las TDIC sobre el currículo escolar, Coll e Illera (2008) destacan la alfabetización digital, que enfoca la adquisición de conocimiento y el desarrollo de las competencias para el dominio funcional de las tecnologías. No obstante a los beneficios que la alfabetización digital ofrece a los estudiantes se observa la necesidad de desarrollar competencias avanzadas que los capacite para mejorar el desempeño respecto al uso de las TDIC (Comisión Económica para América Latina y el Caribe., 2010; Comisión Económica para América Latina y el Caribe., 2011).
En el marco de estas coordenadas, el segundo reto que mencionamos se deriva del primero, pues consiste en poner en marcha prácticas educativas innovadoras que utilicen estrategias diversas para obtener un mejor provecho del potencial de las TDIC para enseñar y para aprender. Análogamente, este reto puede ser pensado en nivel de sistema, de escuela y de aula.

Siguiendo un camino inverso a la discusión anterior, empezamos por pensar en nivel de aula, teniendo en cuenta que constituye un espacio privilegiado en dónde las transformaciones educativas se materializan. Los datos muestran que en lo que concierne a las actividades de aprendizaje mediadas por las TDIC que los profesores llevan a cabo en las escuelas brasileñas, se observa el predominio de los trabajos en grupo y de las clases expositivas (Comitê Gestor da Internet no Brasil., 2016), lo que por sí solo no caracteriza prácticas educativas innovadoras. Por lo que comprendemos, poner en marcha estrategias que utilicen el potencial de las TDIC para enseñar y aprender significa no solo un cambio en el uso de los recursos didácticos, sino en la postura y el rol del profesor en relación a la manera como ocurre el aprendizaje. Así, para constituir una propuesta educativa innovadora, utilizar las TDIC para proponer trabajos en grupo requiere un plan articulado para impulsar el aprendizaje significativo y propositivo de las personas. De hecho, los trabajos en grupo mediados por las TDIC por sí solo no representan innovación, sin embargo, pautar las mismas actividades desde la perspectiva del aprendizaje colaborativa puede resultar una estrategia pedagógica innovadora.

Así, ponemos de manifiesto que no se trata de insertar las TDIC en las prácticas pedagógicas ya instituidas, basta con re-definirlas en función de un referencial teórico que amplíe la mirada sobre las TDIC como instrumentos psicológicos para planificar, regular y orientar los procesos de enseñanza y aprendizaje (Coll, Mauri, \& Onrubia, 2008). En ese aspecto, el debate abierto entre los pares por medio del trabajo interdisciplinar y colaborativo llevado a cabo en la escuela representa un importante soporte y ayuda para los docentes.

Más allá de las cuestiones pedagógicas, en nivel de escuela también son instituidas las reglas formalizadas o no que rigen el uso de las TDIC, como las personas que pueden tener acceso, los espacios habilitados para el uso, las asignaturas que detienen la prioridad, entre otros. Al respecto, retomamos los comentarios anteriores sobre el número reducido de dispositivos TDIC en las escuelas y añadimos que, en general, el uso de la Internet inalámbrica es restringido y no está disponible a los estudiantes (Comitê Gestor da Internet no Brasil., 2016).

Con base a esa realidad, ponderamos que es fundamental ofrecer soporte a las escuelas y universidades a través de las políticas públicas para propulsar la inclusión digital, fomentar la formación continuada y aproximar de la comunidad escolar los servicios de apoyo psicopedagógico. Además, pensamos que la toma de decisiones en el ámbito de las políticas públicas de apoyo a la inclusión digital en la educación debe ser orientada por una mirada que ve el uso de las TDIC en las escuelas como un medio y no como una finalidad en sí misma. Es decir, pensamos que la integración de las TDIC en las escuelas debe ser entendida como una estrategia para lograr mejores niveles de desarrollo educativo y comunitario, lo que significa comprenderlas como herramientas al servicio de un plan pedagógico concreto que promueva el éxito escolar y la mejoría de las condiciones de vida de la población.

El tercer y último reto que mencionamos es crear e implementar políticas públicas que partan de la comprensión de que el aprendizaje no se restringe a las paredes de la escuela. Que las TDIC, especialmente los dispositivos móviles, ofrecen soporte para que las personas sigan aprendiendo en otros espacios más allá de los contextos formales de 
educación. Partiendo de esa mirada, mejorar los procesos educativos por medio de las TDIC pasa por ampliar la red de acceso a Internet en los diversos espacios públicos. Para ello, puede resultar útil actuar en colaboración con otros sectores, como los órganos responsables por la salud y la asistencia social, así como las instituciones privadas, especialmente las organizaciones no gubernamentales (ONG), teniendo en cuenta sumar esfuerzos en beneficio de la comunidad.

En consonancia con todo lo discutido anteriormente, a pesar de considerar importante ampliar el acceso a las TDIC e Internet, asumimos la idea de que eso, por sí solo, no es suficiente para cambiar el desempeño educativo de las personas, toda vez que urge instrumentalizarlas para ir más allá del uso recreativo de las TDIC (Comitê Gestor da Internet no Brasil., 2016). En ese aspecto, resaltamos que la brecha digital en Latinoamérica es observada no solo en relación al acceso de las TDIC por los varios segmentos de la población sino respecto a la manera como las utilizan, lo que significa situarse en niveles diferentes de integración a la cultura digital (González \& Ortiz, 2015).

Por tanto, reiteramos la importancia de las acciones educativas emprendidas dentro y fuera de los contextos formales de educación que enfoquen no sólo la instrumentalización sino también la concientización de las personas respecto al uso responsable y productivo de las TDIC. Por ejemplo, intervenciones educativas que incentiven la búsqueda de contenidos para aprender a través de la red y, además de eso, estimulen la visión crítica acerca del manejo de la información disponible, la legitimidad de las fuentes y para la efectiva construcción de conocimiento y aprendizaje significativo.

\section{Conclusiones}

Al respecto de las diferencias y similitudes observadas en relación a la inclusión digital en Brasil y Colombia, en general, se observa en ambos países la necesidad de crear, implementar y expandir políticas públicas que apoyen la inclusión digital e amplíen las oportunidades educacionales de la población como medio de promover la equidad. En el ámbito de la ampliación de las oportunidades educacionales, reiteramos que los principios de la innovación educativa, tal como concebida en el presente trabajo, ofrece un importante soporte a los procesos de enseñanza y aprendizaje mediados por las TDIC con el ánimo de emprender transformaciones ajustadas al contexto de cada persona y a las necesidades institucionales.

Así, desde los procesos de innovación educativa, destacamos la posibilidad de mejorar los niveles de escolaridad de la población por medio de las TDIC, realizar cursos de formación continuada que ayuden a ampliar las oportunidades laborales, así como empoderar las personas, dentro y fuera de los contextos formales de educación, para promover la autonomía y la construcción de sus propias rutas de aprendizaje a lo largo y ancho de la vida.

Finalmente, otro aspecto que vale la pena destacar es que, en el marco de la brecha digital observada en Brasil y Colombia, los desconectados no se encuentran fuera de los dominios de la sociedad de la información, al final, los efectos de las políticas acarreadas por los cambios económicos llegan para todos en mayor o menor medida. La cuestión de fondo es que, a nuestro juicio, el hecho de no estar integrado a la red ofrece límites muy concretos a las oportunidades de desarrollo en los varios ámbitos, entre ellos, la educación. De modo similar, estar conectado tampoco garantiza sacar provecho de la potencialidad de las TDIC para aprender y mejorar los niveles educacionales. Así, por un lado, consideramos que los procesos de innovación educativa pasan por el acceso y uso de las TDIC dentro y fuera de los contextos formales de educación, y por otro, destacamos que hay que repensar las prácticas y ampliar la mirada acerca de cómo aprenden las personas en el actual contexto de la sociedad del siglo XXI.

\section{Referencias}

Anderson, J., Reder, L., \& Simon, H. (1996). Situated Learning and Education. Educational Researcher, 25(4), 5-11. doi:10.3102/0013189X025004005

Arevalo, M., \& Gamboa, A. (2015). Information and communication technologies within the math syllabus: orientation from educational policies and projects. Panorama, 9(16), 21-30. Obtenido de https://dialnet.unirioja.es/ descarga/articulo/5198903.pdf

Beltran, C. (2016). "Kioscos Vive Digital": a digital strategy to promote the connectivity and social inclusion in rural communities in Colombia. International Journal of Psychological Research, 9(1), 126-130. Obtenido de http://www.scielo.org.co/pdf/ijpr/v9n1/v9n1a12.pdf

Brown, J., Collins, A., \& Duguid, P. (1989). Situated cognition and the culture of learning. Educational Researcher, 18(1), 32-42. doi:10.2307/1176008

Bustamante, J. (2007). Los nuevos derechos humanos: gobierno electrónico e informática comunitaria. Revista Venezolana de Información, Tecnología y Conocimiento(2), 13-27. Obtenido de https://www.redalyc.org/articulo. oa?id $=82340202$

Castells, M. (2000). Toward a Sociology of the Network Society. Contemporary Sociology, 29(5), 693-699. doi:10.2307/2655234

Castells, M., \& Laserna, R. (1989). The new dependency: technological change and socioeconomic restructuring in Latin America. Sociological forum, 4(4), 535-560. Obtenido de https://link.springer.com/article/10.1007/BF01115063

Cebrián, J. (1998). La red. Cómo cambiarán nuestras vidas los nuevos medios de comunicación. Madrid: Taurus.

Coll, C. (2013). La educación formal en la nueva ecología del aprendizaje: tendencias, retos y agenda de investigación. Barcelona, España: Universitat de Barcelona. doi:10.1344/106.000002060

Coll, C., \& Illera, J. (2008). Alfabetización, nuevas alfabetizaciones y alfabetización digital: las TIC en el currículum escolar. Madrid: Ediciones Morata. Obtenido de https://es.calameo.com/books/002073061e43781b096e6

Coll, C., Mauri, T., \& Onrubia, J. (2008). El análisis de los usos reales de las TIC en contextos educativos formales: una aproximación socio-cultural. Revista Electrónica de Investigación Educativa, 10(1), 1-18. Obtenido de https:// redie.uabc.mx/redie/article/view/177/848

Comisión Económica para América Latina y el Caribe. (2010). Panorama Social en América Latina. Santiago de Chile: Publicaciones de las Naciones Unidas. Obtenido de https:// repositorio.cepal.org/bitstream/handle/11362/1236/S2011800_ es.pdf;jsessionid=67075C51CACAE16A4C970E597545271E? sequence $=4$

Comisión Económica para América Latina y el Caribe. (2011). Desafíos para una educación con equidad en América Latina y el Caribe. Encuentro Preparatorio Regional 2011. Naciones Unidas - Consejo Económico y Social. Obtenido de http://www.unesco.org/new/fileadmin/MULTIMEDIA/FIELD/ Santiago/pdf/desafios-Buenos-Aires.pdf

Comitê Gestor da Internet no Brasil. (2016). TIC domicílios 2015: pesquisa sobre o uso das tecnologias da informação e comunicação nos domicílios brasileiros. Sao Paulo: Cetic br. Obtenido de https://cetic.br/media/docs/ publicacoes/2/TIC Dom 2015 LIVRO ELETRONICO.pdf

Comitê Gestor da Internet no Brasil. (2017). Pesquisa sobre o uso das tecnologias de informação e comunicação nas escolas brasileiras: TIC educação 2015. Sao Paulo: Cetic br. Obtenido de https://www.cgi.br/media/docs/ publicacoes/2/tic_edu_2017_livro_eletronico.pdf

Cuevas-Cerveró, A., \& García-Moreno, M. (2010). Ideias, un modelo de evaluación para inclusión digital y alfabetización informacional orientado a salud. Profesional de la Información, 19(3), 240-245. doi:10.3145/epi.2010.may.03

Fernandes, V., Spagnuolo, R., \& Nascimento, E. (2017). Percepção de conselheiros de saúde sobre acesso às informações e inclusão digital. Saúde e Sociedade, 26(1), 218-228. doi:10.1590/s0104-12902017167411

Gonçalves, V., Neris, V., Seraphini, S., Dias, T., Pessin, G., Johnson, T., \& Ueyama, J. (2017). Providing adaptive smartphone interfaces targeted at elderly 
people: an approach that takes into account diversity among the elderly. Universal Access in the Information Society, 16(1), 129-149. doi:10.1007/s10209-015-0429-9

González, J., \& Ortiz, J. (2015). Factores sociodemográficos, educativos y tecnológicos en estadios iniciales de cibercultura en comunidades universitarias. Revista Apertura, 7(2), 1-15. Obtenido de http://wWw. scielo.org. $\mathrm{mx} /$ scielo.php?pid=S1665-61802015000200101\&script=sci abstract

González, M., \& Sánchez, J. (2013). Análisis de las estrategias del Gobierno colombiano para la inclusión de los ciudadanos en la Sociedad de la Información propuestas desde 2000 hasta 2011. Revista de Estudios Sociales, 47, 133-146. doi:10.7440/res47.2013.10

Greene, D. (2016). Discovering the divide: technology and poverty in the new economy. International Journal of Communication, 10, 1212-1231. Obtenido de http://ijoc. org/index.php/ijoc/article/view/3969

Guimarães, L., Ribeiro, J., Echeveste, M., \& Jacques, J. (2013). A study of the use of the laptop XO in Brazilian pilot schools. Computers and Education, 69, 263-273. doi:10.1016/j

Hasebrink, U., Jensen, K., Bulck, H., Hólig, S., \& Maeseele, P. (2015). Changing patterns of media use across cultures: a challenge for longitudinal research. International Journal of Communication, 435-457. Obtenido de https://ijoc.org/ index.php/ijoc/article/viewFile/3452/1303

Instituto Nacional de Geografía Estadística. (abril de 2017). Estadísticas a propósito del día mundial de internet (17 de mayo): Datos Nacionales. Obtenido de http://www.inegi. org.mx/saladeprensa/aproposito/2016/internet2016 0.pdf

Lave, J., \& Wenger, E. (1991). Situated learning: legitimate peripheral participation. Nueva York: Cambridge University Press. Obtenido de https://www.cambridge. org/gb/academic/subjects/psychology/developmentalpsychology/situated-learning-legitimate-peripheral-partic pation?format $=P B \& i s b n=9780521423748$

Leontiev, A. (1974). The problem of activity in psychology. Soviet psychology, 13(2), 4-33. Obtenido de https://doi. org/10.2753/RP01061-040513024

López, P., \& Samek, T. (2009). Inclusión digital: un nuevo derecho humano. Educación y Biblioteca, 114-118. Obtenido de https://eprints.ucm.es/11567/1/INCLUSION DIGITAL\%2C NUEVO DERECHO H.pdf

Martelo, R., Jiménez, I., \& Jaimes, J. (2017). Apoyo al ciudadano desde la extensión universitaria para la accesibilidad e inclusión digital. Formación universitaria, 10(3), 49-60. doi:10.4067/S0718-50062017000300006

Miniwatts Marketing Group. (2018). Internet world stats: usage and population statistics. Obtenido de http://wWw. internetworldstats.com/stats.htm

Moraes, I., Veiga, L., Vasconcellos, M., \& Santos, S. (2009). Inclusão digital e conselheiros de saúde: Uma política para a redução da desigualdade social no Brasil. Ciência e Saúde Coletiva, 14(3), 879-888. doi:10.1590/S141381232009000300023

Observatorio Nacional de las Telecomunicaciones y de la Sociedad de la Información. (2016). La sociedad en red informe anual 2015. Madrid: Sub-Dirección General de Desarrollo Normativo Informes y Publicaciones, Centro de publicaciones. Obtenido de http://www.ontsi.red.es/ontsi/ sites/ontsi/files/Informe\%20Anual\%20La\%20Sociedad\%20 en\%20red\%202015\%20\%28Edici\%C3\%B3n\%202016\%29. pdf

Observatorio Nacional de las Telecomunicaciones y de la Sociedad de la Información. (2017). Dossier de indicadores del índice de economía y sociedad digital.
Madrid: Sub-Dirección General de Desarrollo Normativo Informes y Publicaciones, Centro de publicaciones. Obtenido de http://www.ontsi.red.es/ontsi/

Paixão, P., Freire, V., Lima, M., Linhares, R., Mendonça, A., \& Sousa, M. (2011). A prática de alfabetização em informação e comunicação em saúde: o olhar dos agentes comunitários de saúde sobre o projeto de inclusão digital em Sergipe, Brasil. Interface Comunicação, Saúde, Educação, 18(38), 937-946. doi:10.1590/S1414-32832011000300029.

Pinho-Neto, J. (2012). A inclusão digital dos agentes de limpeza urbana e dos agentes ambientais da coleta seletiva de lixo da cidade de João Pessoa/PB. Informacão e Sociedade: Estudos, 22, 135-143. Obtenido de http://www.periodicos. ufpb.br/ojs/index.php/ies/article/view/13330/8214

Pinho-Neto, J. (2014). Ações de inclusão digital nas penitenciárias no Brasil e na Espanha: um estudo comparativo. Informacão e Sociedade: Estudos, 24(3), 65 80. Obtenido de http://www.periodicos.ufpb.br/ojs/index. php/ies/article/view/18237/12402

Rashid, A. (2016). Digital Inclusion and Social Inequality: gender differences in ICT access and use in five developing countries. Gender, Technology and Development, 20(3), 306-332. doi:10.1177/0971852416660651

Robles, C., Marín, G., \& Zambrano, R. (2012). De la sociedad de la información a la sociedad digital. Web 2.0 y redes sociales en el panorama mediático actual. Revista Faro(15), 1-14. Obtenido de https://idus.us.es/xmlui/bitstream/ handle/11441/29116/Dialnet-DeLaSociedadDeLalnformacio nALaSociedadDigital-4160011.pdf? sequence=1\&isAllowed $=y$

Roth, W.खM., \& Jornet, A. (2013). Situated cognition. WIREs Cognitive Science, 4, 463-478. doi:10.1002/wcs.1242

Said-Hung, E., Fernando, G., Daladier, J., Barreto, R., Ballesteros, B., Vergara, E., \& Ordóñez, M. (2015). Strengthening teaching practices at universities in Colombia through ICT. Educación XXI, 18(2), 277-304. doi:10.5944/educXX1.14605

Santana, C., \& Raymundo, T. (2014). A digital inclusion project for older people: Itinerant mode experience. Gerontechnology, 13(2), 281. doi:10.4017/ gt.2014.13.02.196.00

Selwyn, N. (2004). Reconsidering political and popular understandings of the digital divide. New Media \& Society, 6(3), 341-362. doi:10.1177/1461444804042519

Silva, A. (2017). El impacto de las experiencias de aprendizaje mediadas por las tecnologías digitales de la información y comunicación en la construcción dela identidad de aprendiz. Barcelona: Universidad de barcelona. Obtenido de http://psyed.edu.es/archivos/grintie/Tesis Pereira 2018. $p d f$

Silva, H., Jambeiro, O., Lima, J., \& Brandão, M. (2005). Inclusão digital e educação para a competência informacional: uma questão de ética e cidadania. Ciência da Informação, 34(1), 28-36. Obtenido de http://dx.doi.org/10.1590/s010019652005000100004.

Silva., A. (2011). Educação e tecnologia: entre o discurso e prática. Ensaio: avaliação e políticas públicas em educação. 19(72), 527-554. Campinas. doi:10.1590/5010440362011000400005

Siqueira, A., \& Rothberg, D. (2014). Media education and Brazilian educational policies for the enhancement of learning Comunicar, 113-122. doi:10.3916/C43-2014-11

Vega, O. (2016). De las TIC en la educación a las TIC para la educación. Vector, 11, 24-29. Obtenido de http://vector. ucaldas.edu.co/downloads/Vector11 4.pdf 